\title{
MEDICAL IMAGES AND ITS SECURITY USING CHAOTIC AGORITHM
}

\author{
BANAVATH DHANALAXMI ${ }^{1}$, LAKAVATH SURYANARAYANA ${ }^{2}$, T. SRINIVASULU ${ }^{3}$ \\ ${ }^{1}$ Assistsant Professor, Kakatiya Institute Of Thechnology And Science, Erragattu, Warangal, T.S. \\ ${ }^{2}$ Assistsant Professor, University College Of Pharmaceutical Sciences, Kakatiya University, Warangal, T.S \\ ${ }^{3}$ Professor, Ku College Of Engineering And Technology, Kakatiya University, Warangal, T.S \\ Dhanalaxminov20@mail.com/lakavath_surya@yahoo.com/drstadisetty@gmail.com
}

\begin{abstract}
Medical images are most an important role of many diseases to proper diagnosis. Medical images are essential for many diseases diagnosis, disease progress monitoring, injuries identification, etc. The medical images are recorded by using X-ray images, CT-scan images, MRI- scan images, etc. Therefore, the security of medical images is important and inferior. However, the importance of security of medical images is paramount to avoid mishandling moreover; the conventional cryptographic algorithms are unable to provide robust security. Hence, an innovative algorithm has been developed to provide robust security to medical images to avoid mishandling. Further security improvement for medical image to developed an algorithm of making a simple and effective chaotic system by using a difference of the output sequences of two same existing one-dimension (1D) chaotic maps. Simulations and performance evaluations show that the proposed system is able to produce a one-dimension (1D) chaotic system with better chaotic performances and larger chaotic ranges compared with the previous chaotic maps. The innovative algorithms have been addressed several conventional methods limitation.
\end{abstract}

Key words: Medical images, CT- scan, MRI-scan, chaotic map and Security.

\section{INTRODUCTION:}

It is a well known fact that the medical images are playing an important role in proper diagnosis of many diseases. That is disease diagnosis, disease progress monitoring, injuries identification, etc. The medical images are recorded by using X-ray images, CT-scan images, MRI- scan images, etc. This is basis of digital communicate in security developing in encryption and decryption and optimizing for digitalized content in protective with fast secured and reliable optimizing goals. The medical images have been shown great platform in medical treatment for the patients. These medical images need to be transmitted through public network in order to consult the doctors. Therefore security plays a vital role while transmitting the medical images. The traditional encryption schemes as well as techniques like DES, IDEA, RSA, and AES have not shown better solution in optimizing medical image security for transmission updating. Various researchers have been undergone for medical images encryption in Chaos theory. Medical images encrypts and decryption is a special direction and as undergone gigantic growth with simplicity making in existence and complexity in patient disease diagnosis [1-15].

\section{MEDICAL IMAGE:}

The advancing techniques of nuclear imaging and optical SPP (Single Photon and Positron) ultra sounds, tomography studies and MRI (Magnetic Resonance Imaging), CT-scanner resulted best in identification methods for comparing of digital image sources-image. The modalities helped in patient pathological studies by diagnostic analysis helped in the determination and conformation for patient's disease therapy. And has evolved communicative and computing technology developing various analyzing, transferring, storing and optimizing of data with a requirement of emerged for protection. Regulatory laws have been progressively established and new research topics have emerged to face this problem.

\section{SECURITY ISSUES OF MEDICAL IMAGES:}

The strict rules and ethics legally give protection to patient data and responsible platforms for helping departments for security of health information. [16]. The need to secure medical images and other data on the patient is not only for privacy purposes but also to deter the manipulation that might occur by a malicious person during the transmission from one medical center to another or from one doctor to other doctor. If a medical image is tampered and sent to a specialist or a radiologist, this could lead to a wrong diagnosis that might cause severe problems or leads to the death. The development of medical information system introduces great responsibility for legal protective of health information and studying of individual health data and its acceptability [17]. In the Canada Official Gazette of November 29, 2009, the dissolution of the GIP-CPS (Public Interest Group - Health Professional Card) and were expanded range of missions of the ASIP Santé (Agence Systems Information Partages) [18], a new step were taken in reforming the governance of health information systems. With website 
the ASIP Santé said. Securities vary from one application to another and the privileged aspects they emphasize. Working groups, as those established by the hospital (hospital security working group) or by the CEN/TC 251 Working Group III (for Security, Safety and Quality), show that to meet the security requirement, three characteristics should be ensured: confidentiality, integrity and availability [19].

\section{LITERATURE REVIEW OF CHAOTIC ALGORITHM:}

Chong Ful et.al., [2007] IES (Image Encryption Scheme) by streaming generator for key spacing extending improvising complexity in linear key resulting in restricted precision conditional based on single chaos conversion over scheming[20]. S.Behnia et.al. [2008] A designed algorithm for image encryption with chaotic mapping in 1990 with non linear dynamics shown regime moisturized of systemic chaos encryption determinative [21]. Fuyan Sun et.al. [2008] The chaos based image encryption scheme done with parameters shown developed novel algorithm encryption for chaotic mapping with special processing of larger cycles with expensive for processing speed [22]. Mohammad Ali Bani et.al., [2008] IEA (Image Encryption Approach) used with permutation technique with combination encrypting strong process for image protecting[23]. Xu Shujiang Wang Yinglong et.al., [2009] CIES (Chaos Based Image Encryption Scheme) is proposed based on novel image encrypting with NCM (Non linear Chaotic map) and XOR operation by fixed modifications of wide ranged from starting to ending with modified image[24]. Abhishek Misra et.al., [2011] CBIE (Chaos based Image Encryption Scheme) a cryptographic ranging of schemes chaotic optimizing of analyzing parameters like speed, key space, sensitivity keying, spatial correlation coefficient resulting transforming dynamics with unpredictable behavior and this not enhancing security level of encryption algorithm [25]. Somaya Al-Maadeed et.al., [2012] Image encryption and computational algorithm with wavelet transforming tests of validity proposing algorithm with low sub band of the image. This algorithm of chaotic mapping is not a good diffusing-confusing property [26]. Hazem Mohammad et.al., [2012] This multi chaotic image encryption algorithm using time have proposed new image encryption algorithm with multiple chaotic functions. This enhances encryption algorithm complexity for encrypted systems [27]. F.K.Tabash et.al., [2013] Image Encryption Algorithm with chaotic mapping with 3 logistical mapping and Multi-pseudo random block permutation in proposed image encrypting technique with three steps encrypting image randomized blocks, and generating blocks random permutations. This will be repeated as cycles for iterations with specifying timings [28]. Amit Gupta et.al., [2013] Image Encryption based on nonlinear chaotic conditional algorithm uses tangential power functioning but it has highest disadvantage of larger keying with greatest security level [29]. Chong Fu et.al., [2014] Image cipher with bit level permutation strategy improving approach for image cipher diffusing architecture resulting in secret keying plane image less securitized known plain text attack [30]. Muhammad Asif Gonda et.al., [2015] Substutional for image Encryption Scheme in nonlinear chaotic algorithm proposes IEA secured this information entropy correlation analyzing UACI and NPCR with most wide optimizing value [31]. Usha Salagundi et.al., [2016] Image Encryption Using Scrambling and Diffusion Operation Using Chaotic Map" Scrambling and Diffusion Operation Using Chaotic Map In scrambling stage, input image undergo row scrambling and column scrambling with the help of chaotic map. In diffusion stage manipulating the pixels value based on parity function, simple and easy to implement [32]. Wei Wang et.al., [2016] DWT mapping a new encrypting algorithm with multi chaos characteristics of the deterministic, sensitivity of initial values image is decomposed and special reconstructing by 2 DWT with matrices for more space encryption [33]. Srinivas Koppu et.al., [2017] E-hospitalized and M- hospital encrypt of clinical ultrasound medical image visual encrypts adaptive 2DCM with ultra sound Medical images. The interleaved image is thus transferred over noisy channels was more [34]. Xiao Chen1, et.al.,[2017]. Proposess a new image encryption algorithm based on improved Logistic mapping, Arnold mapping, Kent mapping and wavelet transform. The improved Logistic mapping for pseudo-random number generation and have done xor operation between the pixel value and the key value generated by this method is not expected value improved with a Logistic mapping [35].

\section{ENCRYPTION AND DECRYPTION TECHNIQUES:}

Encryption is defined as data conversions into specific form cipher and not readable by decrypting cipher is converted to previous image or text namely lock-key mechanisms encryption and decryption fig shown in Figure.1.

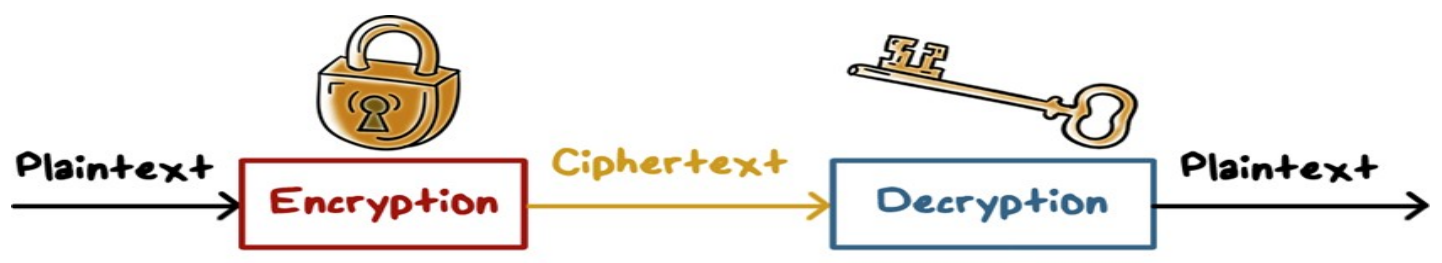

Figure.1 Encryption and Decryption (slideplayer.com) 
Encryption and decryption techniques are very impotent for the medical image security. Cryptography plays an important role in increasing growth of digital data storage and communication. It is used to achieve the mains of security goals like confidentiality, integrity, authentication, non-repudiation. It is also conclude that all the techniques are useful for real-time encryption. Each technique is unique in its own way, which might be suitable for different applications. Many new encryption techniques developing therefore fast and secure standard encryption techniques will always work out with high rate of security.

\section{EXPERIMENTAL SETUP:}

MATLAB deals with integrated visualization of computing and easy programming with easy mathematical platform locations. There are three of matrix laboratory provided with matrix, LINPACK (Linear System Package) and EISPACK (Eigen System Package) for sophisticated matrix software with predeterminative solving with vector formulates. The matrix is fractional time tool boxes of MATLAB with various application devices learning and specialized technology. It is applying for known as ' $m$ ' files for solving various decision and helpful in signal analysis process. The control systems are use for the logic wavelet signaled processing and control systems for the logic simulations etc. MATLAB leads mathematical computation data concerning, modeling, simulations for algorithmic prototyping data analyzing, exploration visualize scientifically, engineering application developing graphical with interface building.

\section{DEVELOPMENT OF MEDICAL IMAGE ENCRYPTION BASED ON ENHANCED 1D CHAOTIC MAP:}

The properties of chaos include deterministic dynamics, unpredictable behavior and non-linear transform. We emphasize the techniques based on chaotic systems, as these systems will improve the security level of encryption algorithm. It is also shown that newly proposed medical image encryption techniques also enhance the security level by introducing more than one chaotic scheme for image encryption algorithms.

The 1D chaotic map have simple buildings, in order that they're being broadly utilized in picture encryption. On this section, three 1D chaotic maps: Logistic, Sine and Chebyshev maps used for our new chaotic procedure will probably be in brief discussed.

Logistic map The logistic map which is an easy dynamic nonlinear equation with problematic chaotic behavior is likely one of the noted 1D chaotic map. It will probably de expressed in the following Equation (1).

$$
x_{n+1}=F_{L}\left(u, x_{n}\right)=u \times x_{n}\left(1-x_{n}\right)
$$

The place $u$ is a control parameter with range of $u \in(0,4]$ and $x_{0}$ is the preliminary worth of chaotic map, $x_{n}$ is the output chaotic sequence.

Sine map: The Sine map is one among 1D chaotic map and has an identical chaotic behavior with the Logistic map. The definition will also be described by means of the following Equation (2).

$$
x_{n+1}=F_{s}\left(r, x_{n}\right)=r \times \sin \left(\pi \bar{x} x_{n}\right)
$$

Where parameter $r \in(0,1]$ and $x_{n}$ is the output chaotic sequence.

Chebyshev map The Chebyshev map is also one of 1D chaotic map and can be described by the following Equation (3).

$$
x_{n+1}=F_{c}\left(a, x_{n}\right)=\cos \left(a \times \arccos x_{n}\right)
$$




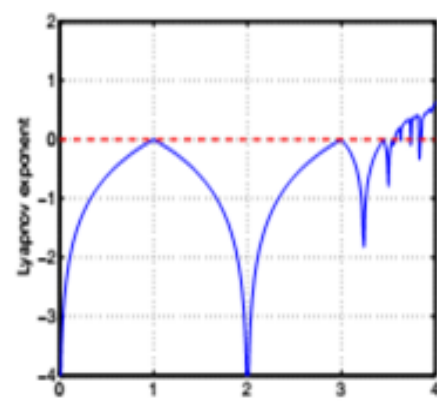

(a)

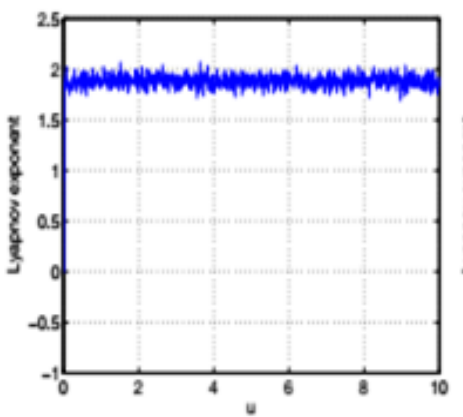

(d)

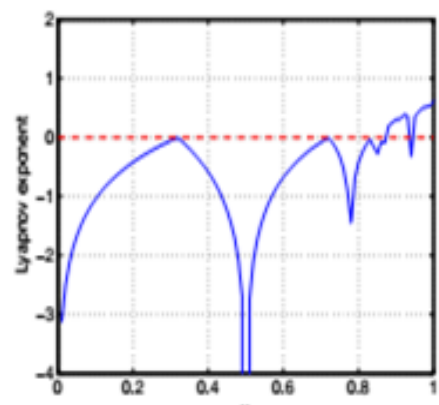

(b)

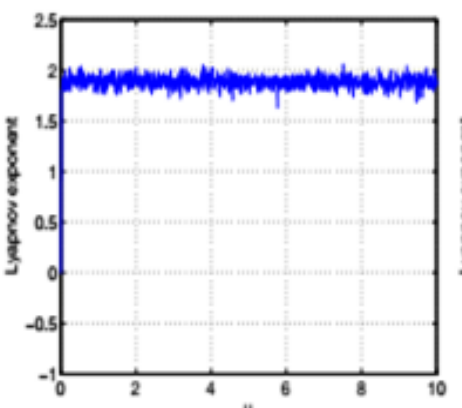

(e)

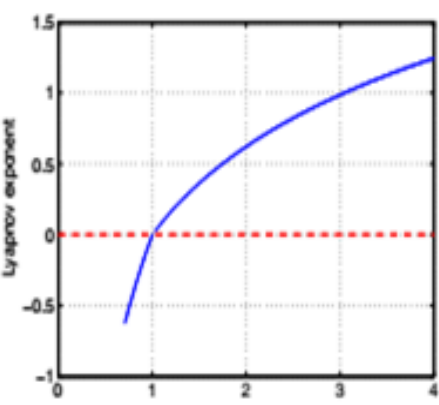

(c)

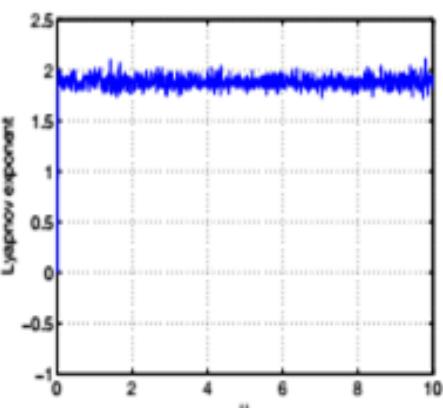

(f)

Figure.2 The Lyapunov exponent diagram of the (a) Logistic map; (b) Sine map; (c) Chebyshev map; (d) LLS; (e) SSS; (f) CCS(sciencedirect.com)

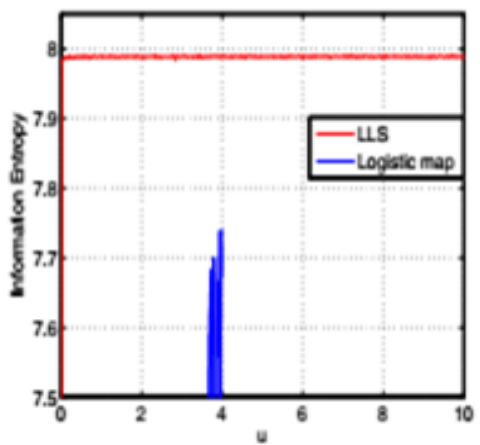

(a)

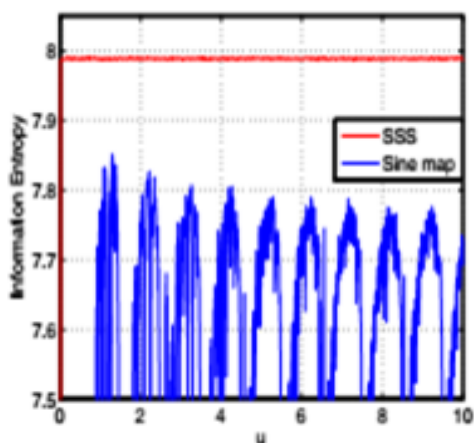

(b)

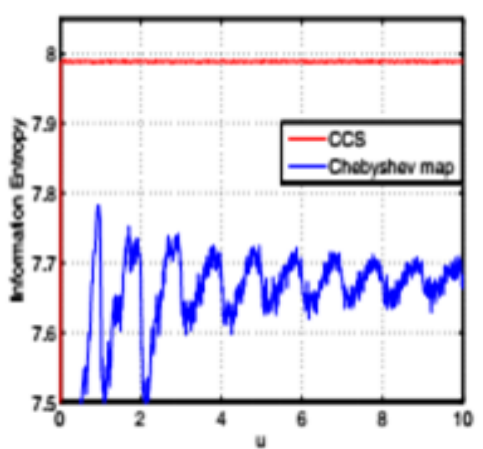

(c)

Figure.3 the Information entropy diagrams of the (a) Logistic map and LLS; (b) Sine map and SSS; (c) Chebyshev map and CCS (sciencedirect.com)

\section{MEDICAL IMAGE ENCRYPTION 1D CHAOTIC ALGORITHM:}

This algorithm is a new Medical image encryption algorithm is proposed and its software in know-how security is tested MATLAB environment. The encryption algorithm makes use of five parameters of ( $\mathrm{x} 0, \mathrm{u}, \mathrm{k}, \mathrm{N}_{0}, \mathrm{lp}$ ) because the safety key. The diagrams of the proposed cryptosystem are shown in Figure.4.

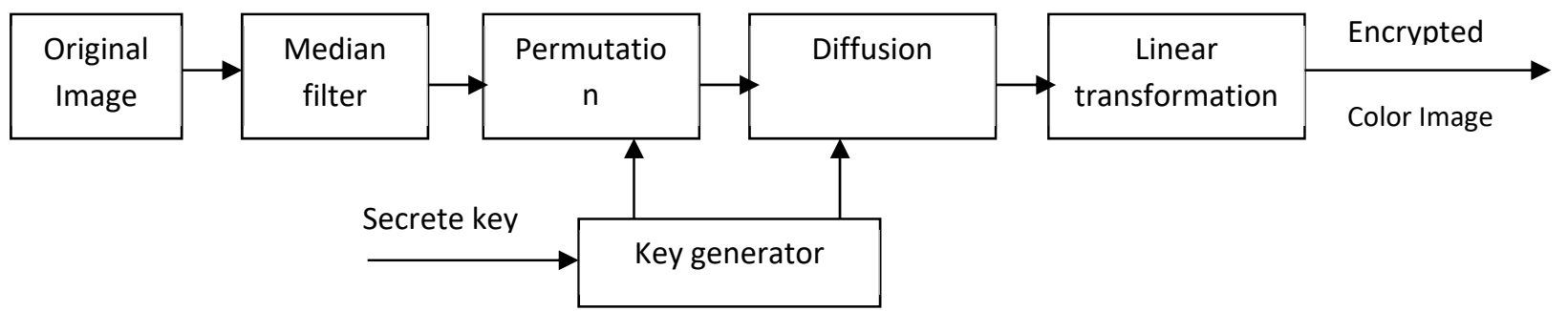

Figure.4 The block diagram of the proposed cryptosystem 


\section{ENCRYPTION PROCESS OF MEDICAL IMAGE:}

The color image with the size of $\mathrm{M} \times \mathrm{N}$ is divided into 3 images with $\mathrm{R}, \mathrm{G}$ and $\mathrm{B}$ channels respectively, and then the 3 images are linked to make a grayscale image with the size of $\mathrm{M} \times 3 \mathrm{~N}$. In the case of the Grayscale image with the size of $\mathrm{M} \times \mathrm{N}$, it will be used without conversion. A median filter is used to remove noise from medical images because noise is always available with medical images.

\section{ENCRYPTION PROCESS:}

Step 1: The colour medical image with the dimensions of $\mathrm{M} \times \mathrm{N}$ is divided into three snap shots with $\mathrm{R}, \mathrm{G}$ and $\mathrm{B}$ channels respectively, after which the 3 photos were linked to make a grayscale photo with the dimensions of $\mathrm{M}$ $\times 3 \mathrm{~N}$. Within the case of the Grayscale snapshot with the dimensions of $\mathrm{M} \times \mathrm{N}$, it will be used without conversion.

Step 2: A median filter is used to remove noise from medical images because noise is always available with medical images.

Step 3: The grayscale image obtained above is converted into the 1D image pixel matrix

$$
\mathrm{P}=\left\{\mathrm{p}_{1}, \mathrm{p}_{2}, \ldots \mathrm{p}_{\mathrm{M} \times 3 \mathrm{~N}}\right\} \text { with the size of } \mathrm{M} \times 3 \mathrm{~N} \text {. }
$$

Step 4: The chaotic sequence $\mathrm{X}$ used in the encryption system is obtained in the above-mentioned new chaotic system. Where $\mathrm{x}_{0}, \mathrm{u}$ and $\mathrm{k}$ are initial values of the chaotic system and are used as the security keys. Iterate the new chaotic system $\left(\mathrm{M} \times 3 \mathrm{~N}+\mathrm{N}_{0}\right)$ times and discard the former $\mathrm{N}_{0}$ elements to make a new sequence with $\mathrm{M} \times 3 \mathrm{~N}$ elements. Where $\mathrm{N}_{0}$ is a constant used as the security key.

Step 5: Obtain the permutation position matrix $\mathrm{X}^{\prime}=\left\{\mathrm{X}^{\prime}{ }_{1}, \mathrm{x}^{\prime}{ }_{2} \ldots \mathrm{x}^{\prime}{ }_{\mathrm{M} \times 3 \mathrm{~N}}\right\}$ by sorting the chaotic sequence $\mathrm{X}$ in ascending order. The process is shown in Figure.6.5 [a].

Step 6: Obtain the permuted Image pixel matrix $\mathrm{P}^{\prime}=\left\{\mathrm{p}^{\prime}{ }_{1}, \mathrm{p} /{ }_{2}, \ldots \mathrm{p}^{\prime}{ }_{\mathrm{M} \times 3 \mathrm{~N}}\right\}$ by using the permutation position matrix $\mathrm{X}^{\prime}$ and the Image pixel matrix P. Permutation Equation(4) can expressed by the following.

$$
P^{\prime}(i)=P\left(X^{\prime}(i)\right) \text {; }
$$

Step 7: Obtain the diffusion matrix $\mathrm{D}^{\prime}=\left\{\mathrm{d}^{\prime}{ }_{1}, \mathrm{~d}^{\prime}{ }_{2}, \ldots \mathrm{d}^{\prime}{ }_{\mathrm{M} \times 3 \mathrm{~N}}\right\}$ by the following Equation(5).

$$
D^{\prime}(i)=\bmod \left(f \operatorname{loor}\left(X(i) \times 10^{14}\right), 256\right. \text {; }
$$

Step 8: Obtain the encrypted Image pixel matrix $\mathrm{C}=\left\{\mathrm{c}_{1}, \mathrm{c}_{2}, \ldots \mathrm{c}_{\mathrm{M} \times 3 \mathrm{~N}}\right\}$ from the diffusion matrix $\mathrm{D}^{\prime}$ and the permuted Image matrix $\mathrm{P}^{\prime}$ by the following diffusion Equation(6).

$$
C(i)=\bmod \left(P^{\prime}(i) \oplus D^{\prime}(i), 256\right) \otimes C(i-1) ;
$$

Where $\oplus$ is the arithmetic plus operator, $\oplus$ bit-level XOR operator, and C (i-1) the previous encrypted pixel. The process is shown in Figure.6.5 (b).

The step 8 not only avoids the repetition of linear (permutation)-nonlinear (diffusion) conversion to shorten the encryption time, but also increases the strength of encryption.

Step 9: Apply Histogram equalization for encrypted Image to get improved encrypted Image.

Step 10: Getting a new encrypted medical image pixel matrix $\mathrm{c}^{\prime}=\left(\mathrm{c}^{\prime}{ }_{1}, \mathrm{c}^{\prime}{ }_{2}, \ldots \ldots . \mathrm{c}_{\mathrm{M} \times 3 \mathrm{~N}}\right)$ by rotating the above obtained matrix $C$ to the left by the amount of $l p$. Where $l p$ is used as a security key and $l p \in[1, M \times 3 N]$.

The new image pixel matrix $\mathrm{C}^{\prime}$ is obtained in the following Equation (7).

$$
\begin{cases}C^{\prime \prime}(i-l p)=C(i) ; & i-l p \geq 1 \\ C^{\prime \prime}(i-l p)+M \times 3 N=C(i) ; & i-l p \geq 1\end{cases}
$$

Step 11: Convert the $C^{\prime}$ into the $R, G$ and $B$ color Image with the size of $M \times N$.

\section{DECRYPTION PROCESS:}

The decryption is the inverse process of encryption. The permutation and diffusion equations used in decryption are as follows Equation (8) and (9).

$$
\begin{gathered}
P\left(X^{\prime}(i)\right)=P^{\prime}(i) ; \\
P^{\prime}(i)=\bmod \left(C(i) \otimes C(i-1)-D^{\prime}(i), 256\right) ;
\end{gathered}
$$

Where '-' is the arithmetic minus operator. The process of the Equation (9) is shown in Figure.6.5[c]. The encryption and decryption algorithms are simple, but they are enough to increase the strength of encryption. They can be applied not only to color image, but also to grayscale image.

\section{ANALYSIS AND RESULTS:}

To assess the performance of the encryption algorithm, we made a simulation test with MATLAB 2013a. The above proposed SSS and color image with the dimensions of $256 \times 256$ are used. The initial worth of SSS $x_{0}=$ 0.456 , the manage parameter $\mathrm{u}=5.4321, \mathrm{k}=14$ and $\mathrm{N}_{0}=1000$. The outcome of encryption and decryption are 
shown in Figure 5. This indicates that every one encrypted photos are noise-like ones and may also be efficiently utilized to pix of various forms comparable to grayscale photos, color photos and binary pictures.

\section{SAFETY KEY SPACE:}

Space for higher protection performance, the encryption algorithm will have to be very sensitive to any change of its security key and have a larger area than 2100 , sufficient to withstand the brute drive attack. Our encryption algorithm has 5 protection keys: $\mathrm{u}, \mathrm{x}_{0}, \mathrm{k}, \mathrm{N}_{0}$, lp. Where $\mathrm{u} \in(0,10], \mathrm{x} 0 \in(0,1]$, ok $\in[8,20], \mathrm{lp} \in[1, \mathrm{M} \times 3 \mathrm{~N}]$. Here we compute the $u$ and $\mathrm{x}_{0}$ within the accuracy of $10^{-16}$, set the size of photo to $256 \times 256$, set $\mathrm{N}_{0}=10^{3}$ and remember the $k$, a good way to get the total key space as $10^{16} \times 10^{16} \times(256 \times 256 \times$ three $) \times 10^{3} \times 12 \approx 2^{138}$. Which means our algorithm can withstand the brute drive assault.

\section{HISTOGRAM EVALUATION IMAGE:}

Histogram displays the distribution of pixel values of a snapshot. To resist statistic assaults, the photo histogram should be flat. Figure.6.9, show the Histogram of MRI Image for knee joint of leg photographs and the histograms of their encrypted photos. As proven in Figure. 11, the Histogram of CT Scan of chest Image encrypted snapshot has a just right uniform distribution, in order that it's adequate to resist statistic assaults. The same Histogram result was seen in the Figure.9 MRI image for knee joint of leg, Figure.12 CT Scan of chest image, Figure.15 CT scan GIT, Figure.18 CT scan of lung, and Figure.21MRI scan of the brain.

\section{CORRELATION OF TWO ADJACENT PIXELS:}

Picture data commonly has some intrinsic features, corresponding to high redundancy of information and powerful correlation amongst neighboring pixels, and it may be utilized by attackers for attacking expertise. Within the test we randomly selected a thousand pairs of adjacent pixels from the original photographs and the encrypted photographs and analyzed the correlations at horizontal, vertical and diagonal guidelines Table2. Table 1is the MSE and PSNR value shown better result of Figure.10 MRI image for knee joint of leg, Figure.13 CT Scan of chest image, Figure.16 CT scan GIT, Figure.19 CT scan of lung, and Figure.22 MRI scan of the brain.

\section{DIFFERENTIAL ANALYSIS OF MEDICAL IMAGE:}

In the $1 \mathrm{D}$ chaotic algorithm is very specific for medical images, in order to test the effect of a pixel change on the entire cipher image, present work is usually compared with existing work: the Number of Pixels Change Rate (NPCR) and the Unified Change Intensity (UACI). Table 2: lists the medical Image of NPCR and UACI values. As can be seen from Table 2: different values of present and existing work encryption, the NCPR value very close to 1 and UACI value close to 0 .

\section{PSNR AND MSE VALUE CALCULATION}

The restoring ability of a medical image is evaluated by PSNR and MSE expressed in the following Equation (10).

$$
\begin{gathered}
\mathrm{PSNR}=10 \times \log \left(\frac{55^{2}}{M S E}\right. \\
\mathrm{MSE}=\frac{1}{M \times N} \sum_{i=1}^{N} \sum_{j=1}^{M}\left[I(i, j)-I^{\prime}(i, j)\right]^{2}
\end{gathered}
$$

TIME:

Time period is significant change in this algorithm between in existing and present work, Table 2: gave the time periods of different medical images.

Table 1: MSE and PSNR results of existing and present work

\begin{tabular}{|c|c|c|c|c|}
\hline \multirow{2}{*}{ IMAGES } & \multicolumn{2}{|c|}{ Existing work } & \multicolumn{2}{c|}{ Present work } \\
\hline & MSE & PSNR & MSE & PSNR \\
\hline MRI of knee joint & 44.41970 & 31.65510 & 21.08691 & 34.8906 \\
\hline CT scan of chest & 20.26566 & 35.09756 & 11.7235 & 37.4402 \\
\hline CT Scan GIT & 56.8926 & 30.5802 & 32.26502 & 33.0435 \\
\hline CT Scan lung & 49.7853 & 31.1598 & 38.21612 & 32.3083 \\
\hline MRI Scan brain & 61.7437 & 30.2249 & 44.39152 & 31.6578 \\
\hline
\end{tabular}


Table 2: Correlation, NPCR and UACI values of existing and present work

\begin{tabular}{|l|l|l|l|l|l|l|l|l|l|l|}
\hline Images & \multicolumn{5}{|c|}{ Existing work } & \multicolumn{5}{c|}{ Present work } \\
\hline & $\begin{array}{l}\text { Time } \\
(\mathbf{s e c})\end{array}$ & $\begin{array}{l}\text { Vertical } \\
\text { correlation }\end{array}$ & $\begin{array}{l}\text { Horizontal } \\
\text { correlation }\end{array}$ & NPCR & UACI & Time & $\begin{array}{l}\text { Vertical } \\
\text { correlation }\end{array}$ & $\begin{array}{l}\text { Horizontal } \\
\text { correlation }\end{array}$ & NPCR & UACI \\
\hline 1 & 6.88 & 0.02479 & 0.59026 & 0.99609 & 0.33463 & 0.42 & -0.0605 & 0.533729 & 0.99609 & 0.33463 \\
\hline 2 & 7.47 & -0.53907 & 0.15140 & 0.99608 & 0.33463 & 0.43 & -0.1016 & 1.8558 & 1.0000 & 0.33650 \\
\hline 3 & 6.92 & 0.3765 & 0.18440 & 0.99909 & 0.13042 & 0.93 & 0.4508 & 0.149800 & 0.73492 & 0.30180 \\
\hline 4 & 7.95 & 0.3601 & 0.24027 & 0.99982 & 0.15158 & 0.94 & 0.3564 & 0.231770 & 0.58935 & 0.18376 \\
\hline 5 & 7.32 & 0.6177 & 0.09086 & 0.99994 & 0.23428 & 1.05 & 0.6066 & 0.080420 & 0.61598 & 0.24642 \\
\hline
\end{tabular}

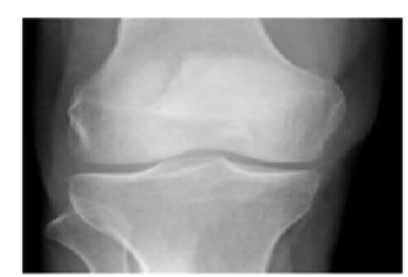

MRI knee joint Image

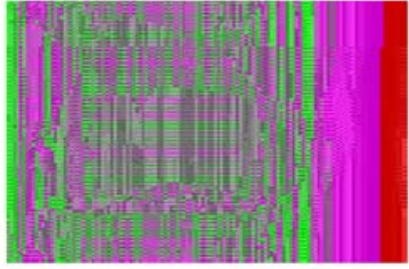

Encrypted Image

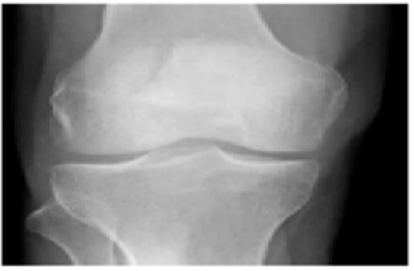

Decrypted Image

Figure.5 Previous work of MRI Image for knee joint of leg plaintext and cipher image

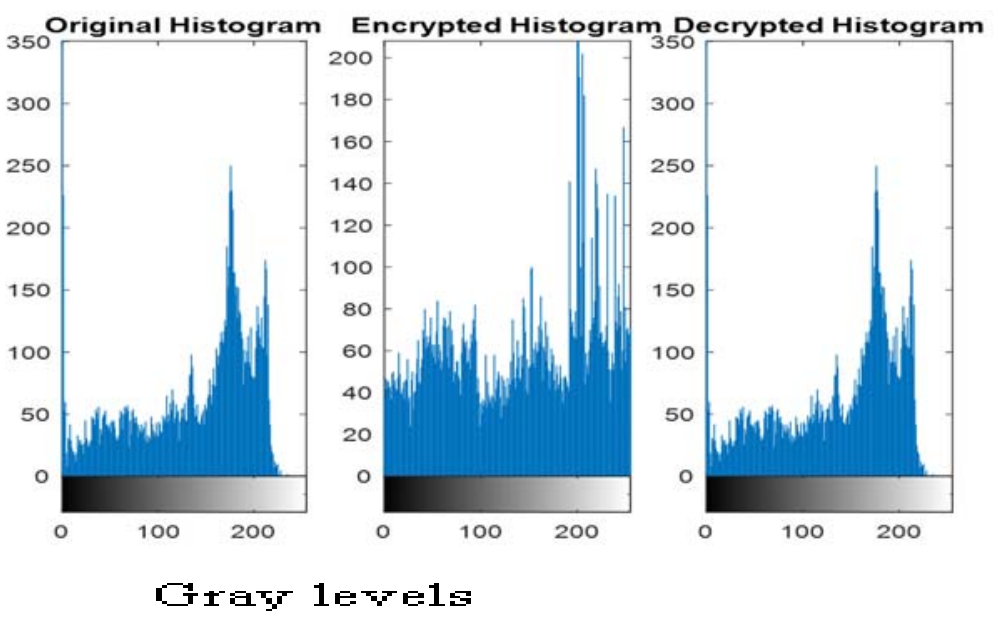

Figure.6 Previous work of Histogram of MRI image for knee joint of leg

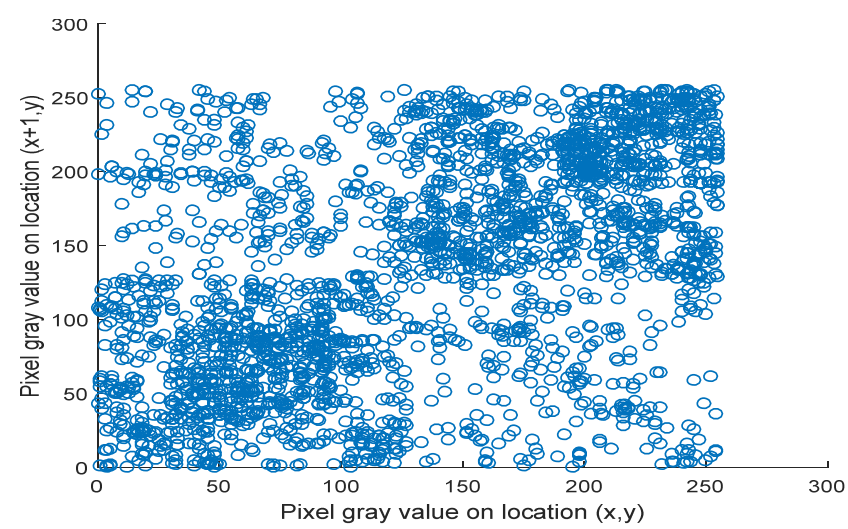

Figure.7 Previous work of Correlation plot of MRI image for knee joint of leg 

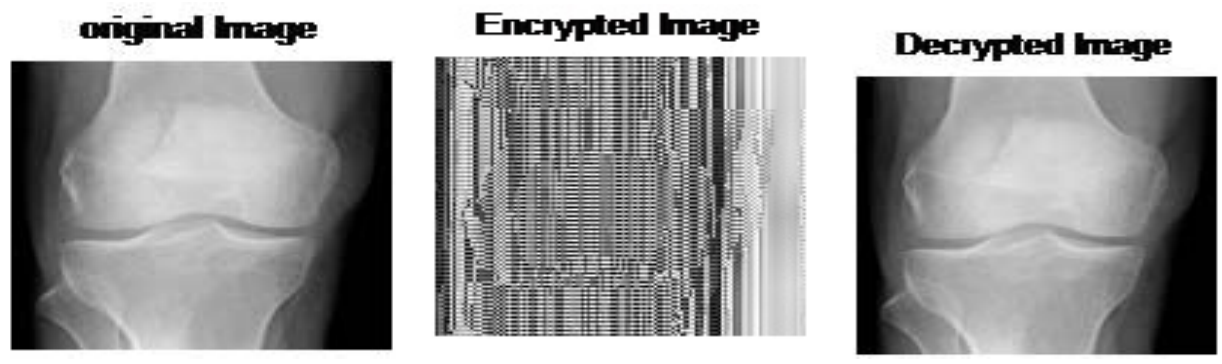

Figure.8 Present work of MRI Image for knee joint of leg plaintext and cipher image
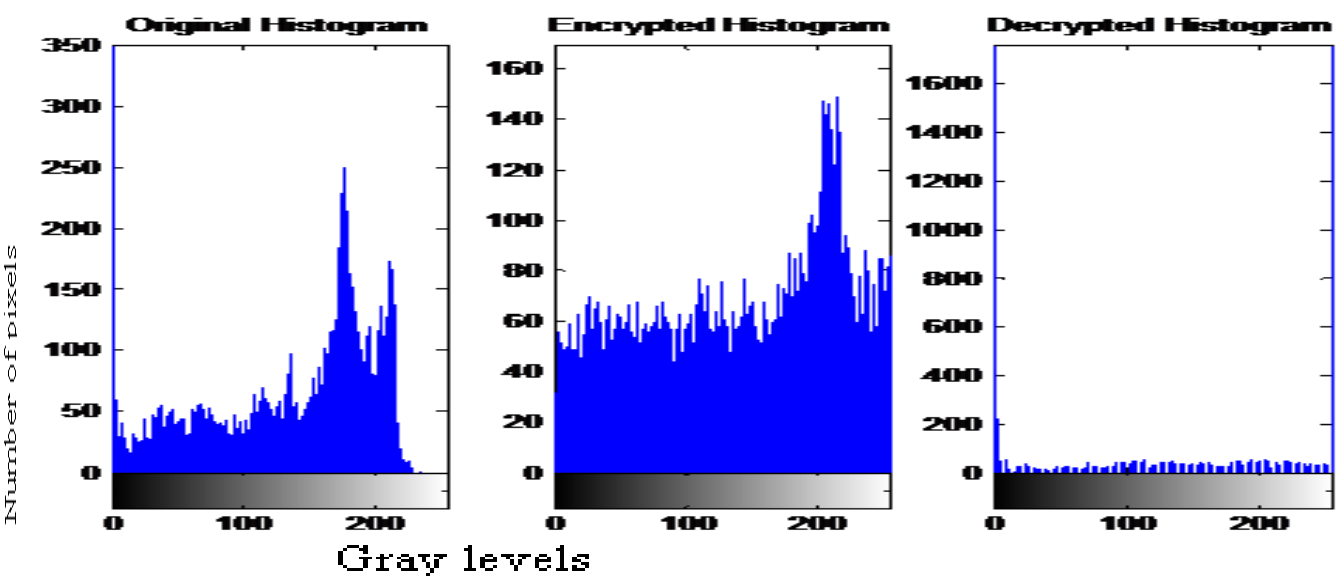

Figure.9 Present work of Histogram of MRI image for knee joint of leg

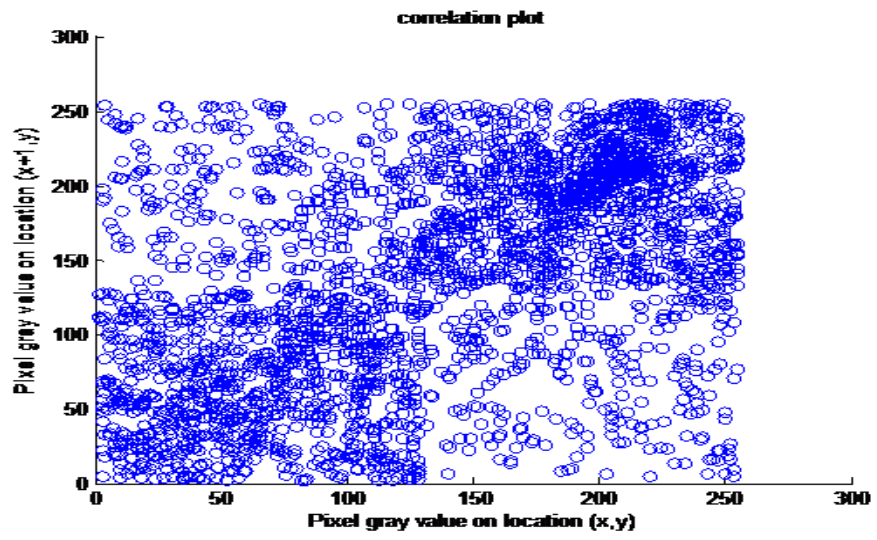

Figure.10 Present work of Histogram of MRI image for knee joint of leg

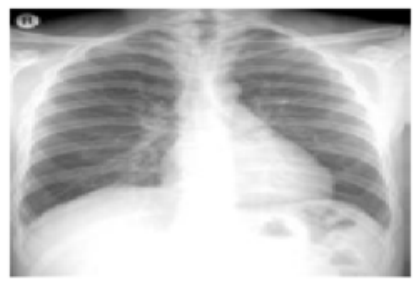

Original CT scan of chest Image

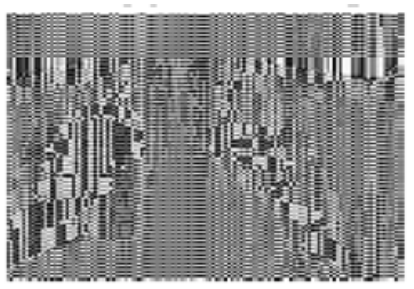

Encrypted chest Image

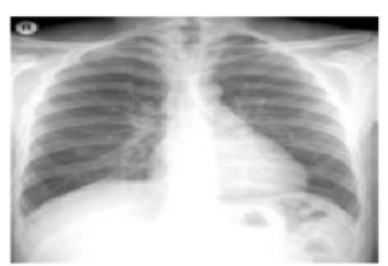

Decrypted chest Image

Figure.11 CT Scan of chest Image plaintext and cipher image 

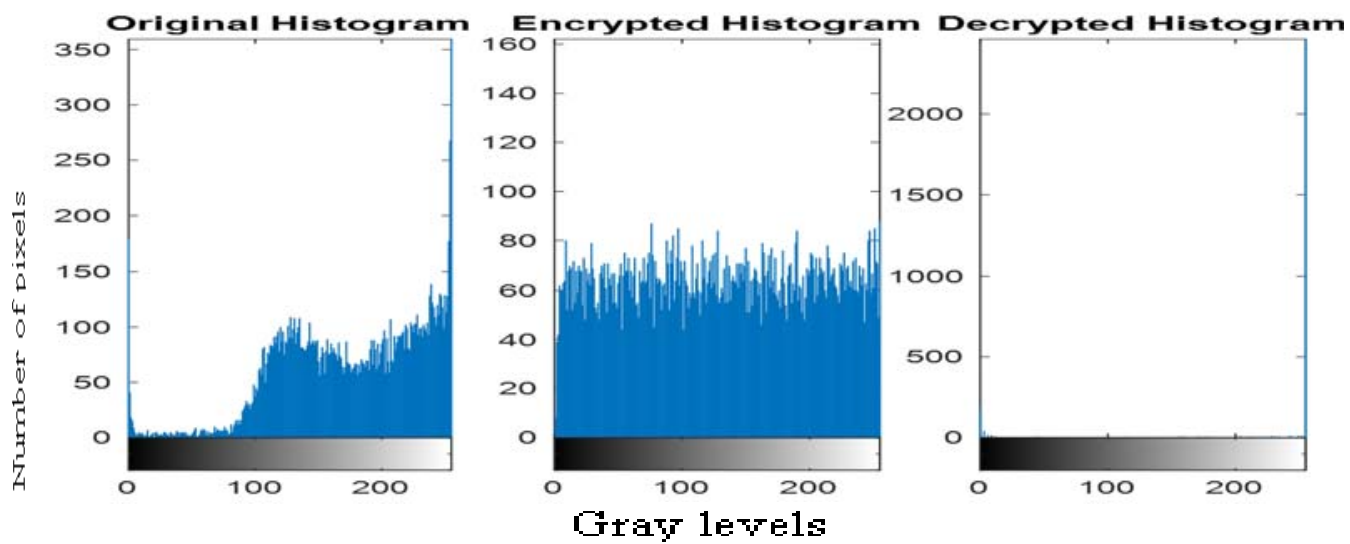

Figure.12 Histogram of CT scan of chest image

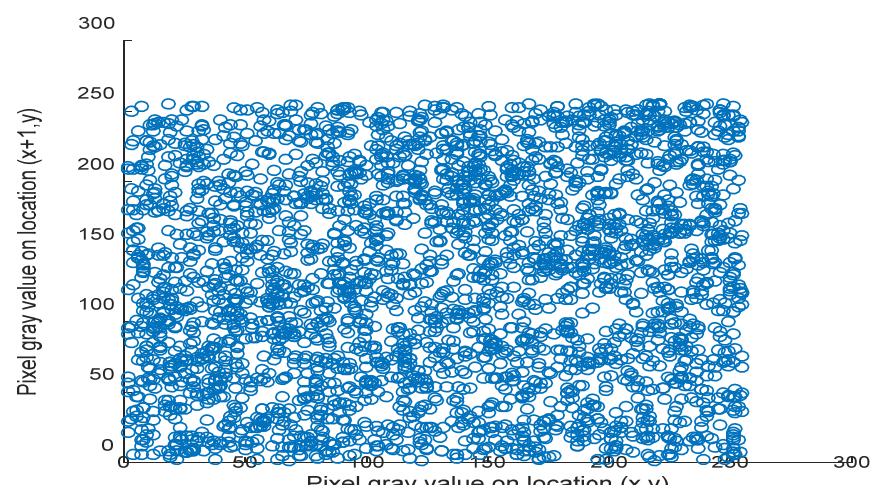

Figure.13 Correlation plot CT scan of chest image
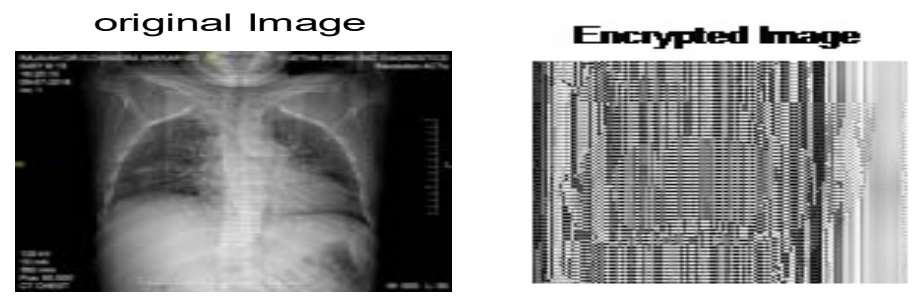

Decrypted Image

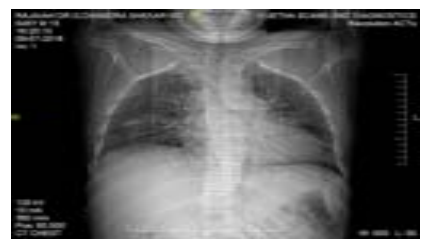

Figure.14 CT scan GIT of plaintext and cipher text and decrypted image
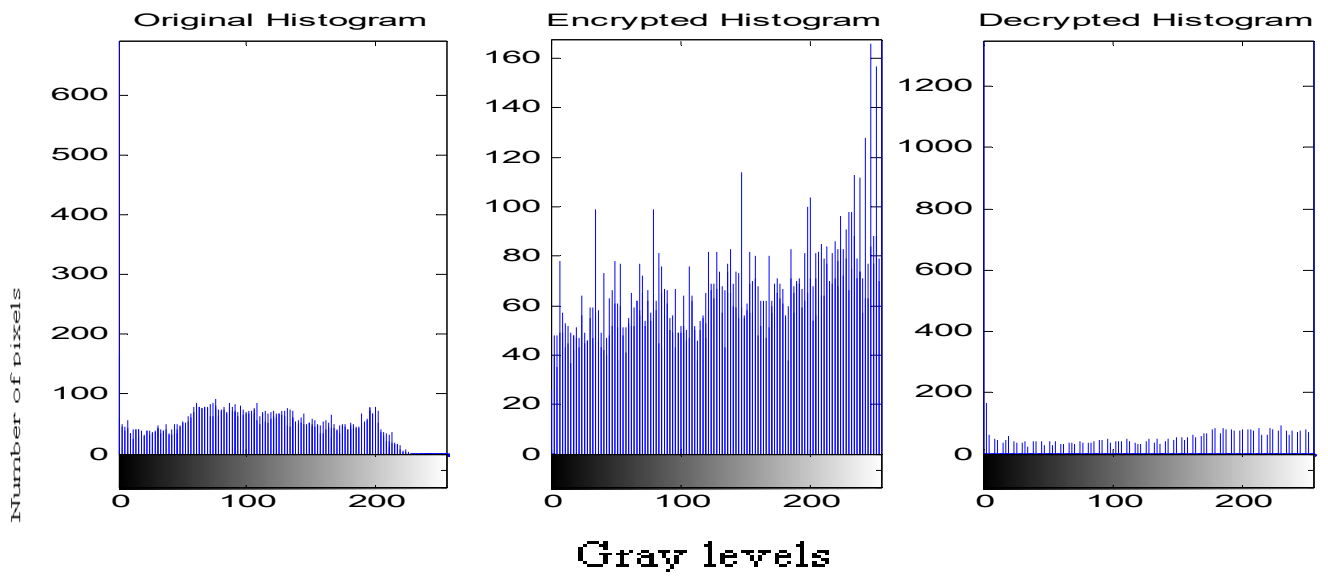

Figure.15 CT Scan GIT histogram of plaintext and cipher text and decrypted image 


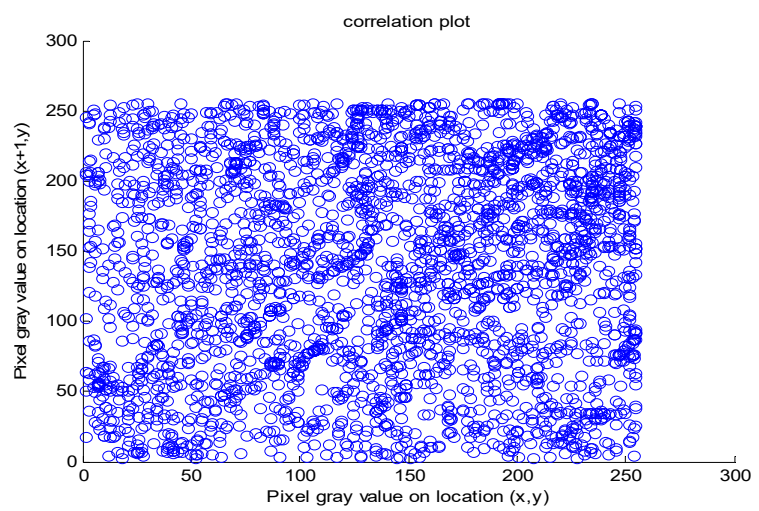

Figure.16 CT scan GIT correlation plot of plaintext and cipher text and decrypted image
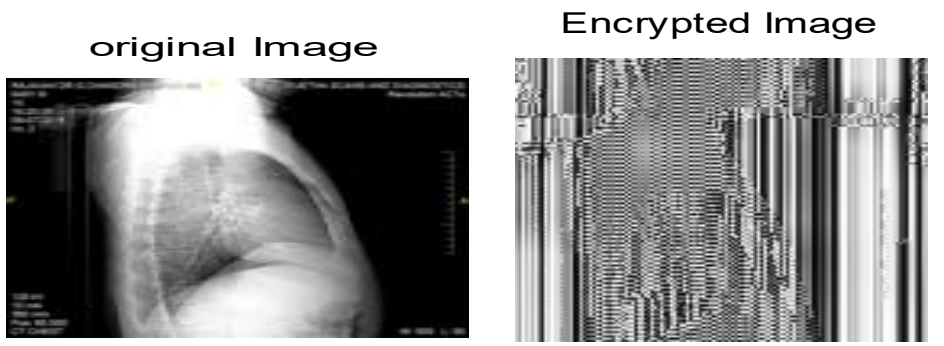

\section{Decrypted Image}

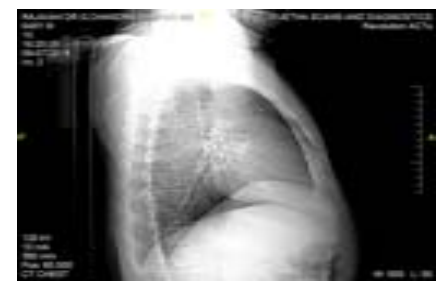

Figure.17 CT scan lung of plaintext and cipher text and decrypted image
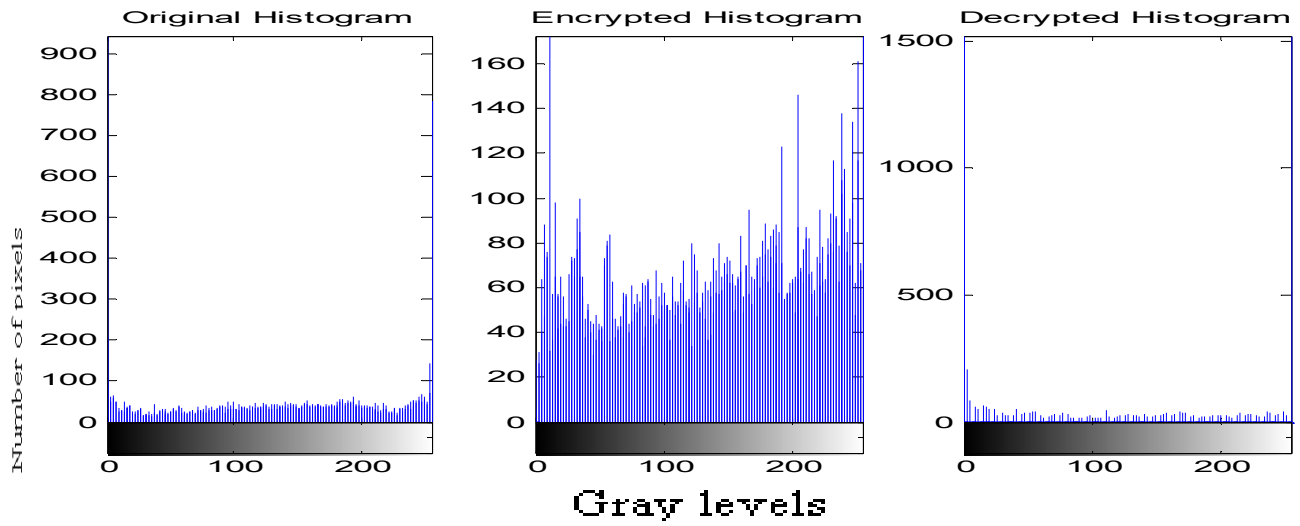

Figure.18 CT scan lung histogram of plaintext and cipher text and decrypted image

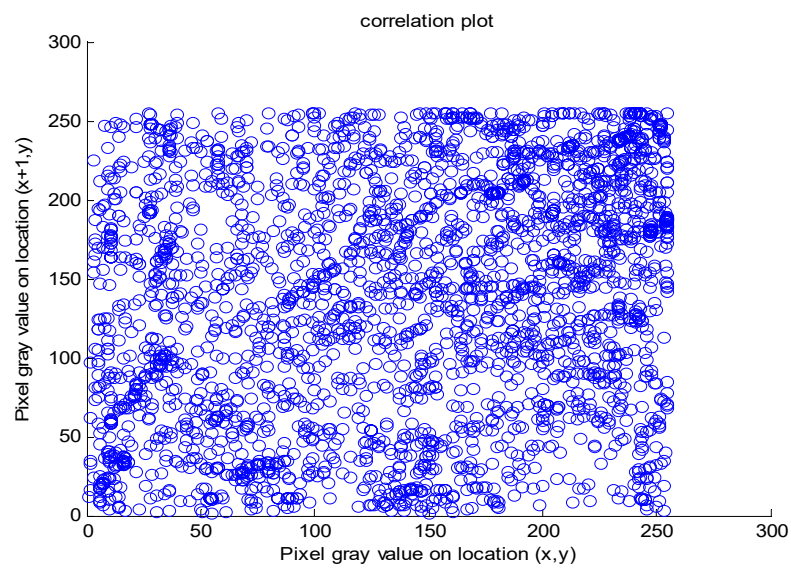

Figure.19 CT scan lung correlation plot of plaintext and cipher text and decrypted image 

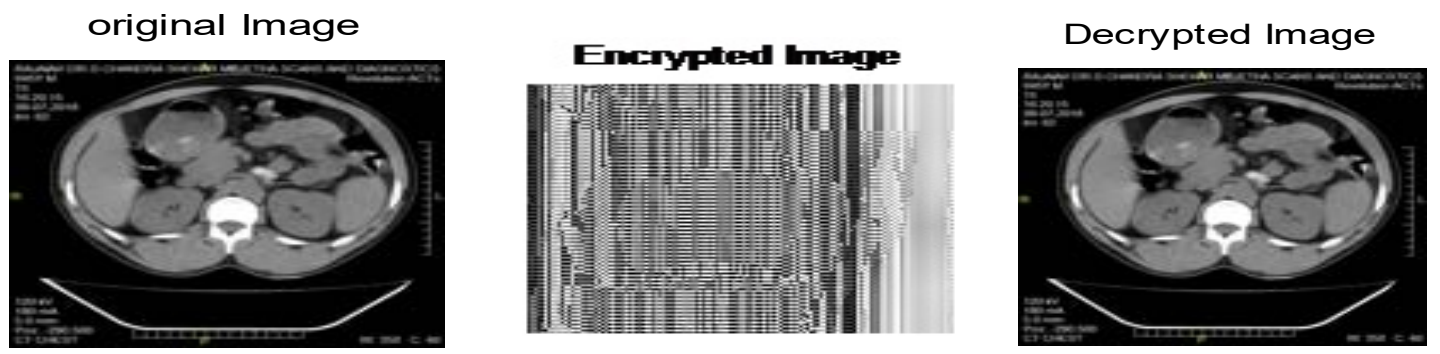

Figure.20 MRI Scan brain of plaintext and cipher text and decrypted image
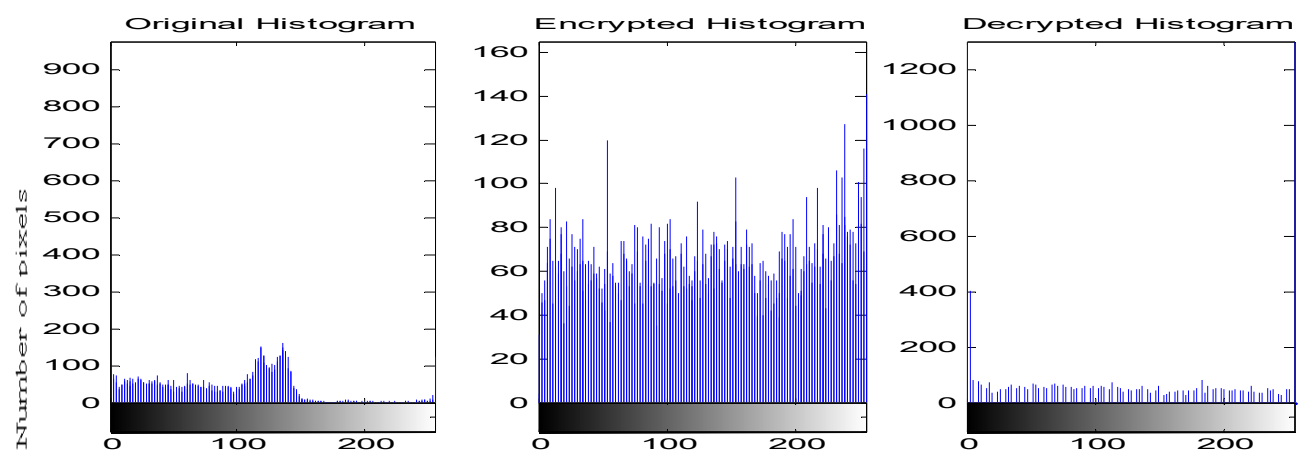

Gray lewels

Figure.21 MRI Scan brain histogram of plaintext and cipher text and decrypted image

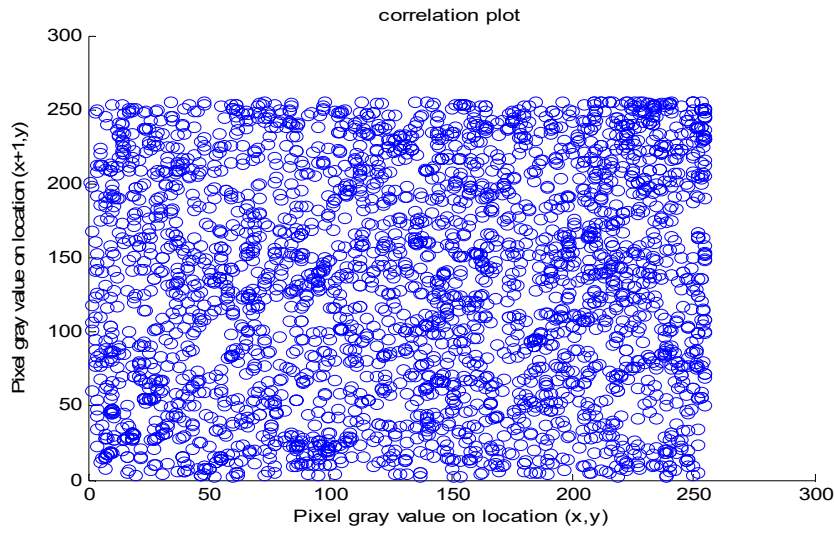

Figure.22 MRI Scan brain correlation plot of plaintext and cipher text and decrypted image

CONLUSION:

The results of the algorithm are efficient when compared present work with previous work. In Table1: MSE value is decrease and PSNR value is increased. The better result of algorithm MSE value is low and PSNR value is high. Table 2: NPCR value is near to 1 and UACI value is near to 0 . Therefore, modified $1 \mathrm{D}$ chaotic algorithm is gave better results and also predicts of algorithm is efficient for medical image security. Major fact find from this algorithm are this algorithm is robustness and high secure for medical image, clarity of medical image encryption and decryption is more, time period is very low when compared to other algorithm and statistical values of this algorithm almost equal to standard statistical values.

\section{REFERENCES:}

[1] G. Chen, X.Y. Zhao, “A self-adaptive algorithm on image encryption”, International Journal Software, 16, 1987, pp. $1975-1982$.

[2] N. Bourbakis, C. Alexopoulos, "Picture data encryption using SCAN patterns", Pattern Recognition, 25(6), 1992, pp. 567-581.

[3] K.L. Chung, L.C. Chang, "Large encrypting binary images with higher security", Pattern Recognition Letters, 19(5-6), 1998, pp. 461468.

[4] J.C. Yen and J.I. Guo, "A new image encryption algorithm and its VLSI architecture", IEEE Workshop on Signal Processing System, 3 1999, pp. 430-437.

[5] 5. H. Cheng, X.B. Li, "Partial encryption of compressed images and videos", IEEE Transaction in Signal Processing, 48(8), 2000, pp. 2439-2451.

[6] J. Fridrich, "Symmetric ciphers based on two-dimensional chaotic maps", International Journal of Bifurcation and Chaos, 8(6), 1998, pp.1259-1284.

[7] G. Alvarez, Shujun Li, "Crypt analyzing a nonlinear chaotic algorithm (NCA) for image encryption", Commun Nonlinear Sci. Numer.Simulation, 14, 2009, pp. 3743-3749. 
[8] M.S. Baptista, "Cryptography with chaos", Physics Letter A, 240(1-2), 1999, pp. 50-54.

[9] X. Liao, X. Li, J. Pen and G. Chen, "A digital secure image communication scheme based on the chaotic chebyshev map", International Journal of Communication Systems, 17(5), 2004, pp. 437-445.

[10] F. Han, X. Yu and S. Han, "Improved baker map for image encryption", International Symposium on Systems \& Control in Aerospace \&Astronautics, 2, 2006, pp. 1273-1276.

[11] S. Lian, J. Sun, Z. Wang, "A block cipher based on a suitable use of chaotic standard map", Chaos Solitons \& Fractals, 26(1), 2005, pp.117-129.

[12] B. He, F. Zhang, L. Luo, M. Du, Y. Wang, “An image encryption algorithm based on spatiotemporal chaos”, International Congress on Image and Signal Processing, 2009, pp. 1-5.

[13] G. Chen, Y. Mao, C.K. Chui, "A symmetric image encryption schemes based on 3D chaotic cat maps", Chaos Solitons and Fractals, 21(3) 2004, pp. 749-761.

[14] Z.H. Guan, F. Huang, W. Guan, "Chaos-based image encryption algorithm”, Physics Letters A, 346(1-3), 2005, pp. $153-157$.

[15] T. Gao, Z. Chen, "Image encryption based on a new total shuffling algorithm", Chaos Solitons \& Fractals, 38(1), 2008, pp. 213-220.

[16] H. Nyeem, W. Boles, and C. Boyd, "A review of medical image watermarking requirements for teleradiology," Journal of Digital Imaging, vol. 26, no. 2, pp. $326-343,2013$.

[17] J. G. Hodge, L. O. Gostin, and P. D. Jacobson, "Legal Issues Concerning Electronic Health Information: Privacy, Quality, and Liability," JAMA, vol. 282, no. 15 , pp. 1466-1471, Oct. 1999

[18] "Réforme de la gouvernance des systems d'information de santé." Communiqué de Presse, 03- Dec-2009.

[19] Chong Fu1, Zhen-chuan Zhang1, Ying Chen2, and Xing-wei Wang1, "An Improved Chaos- Based Image Encryption Scheme" lecture notes in computer science2007.

[20] 20. S. Behnia, A. Akhshani, H. Mahmodi, A. Akhavan "A novel algorithm for image encryption based on mixture of chaotic maps". Chaos, Solitons and Fractals 35 (2008), pp 408-419.

[21] Fuyan Sun a, Shutang Liu a, Zhongqin Li , Zongwang Lu" "A novel image encryption scheme based on spatial chaos map" Chaos, Solitons and Fractals 38 (2008) 631-640.

[22] Mohammad Ali Bani Younes and Aman Jantan, "An Image Encryption Approach Using a Combination of Permutation Technique Followed by Encryption" IJCSNS International Journal of Computer Science and Network Security, VOL.8 No.4, April 2008.

[23] Xu Shujiang Wang Yinglong, Guo Yucui Wang Cong, "A Novel Chaos-based Image Encryption Scheme” International Conference on Information Engineering and Computer Science 2009.

[24] Abhishek Misra, Ashutosh Gupta, Damodar Rai “Analysing the Parameters of Chaos Based Image Encryption Schemes" World Applied Programming (WAP), Vol (1), No (5), December 2011

[25] Somaya Al-Maadeed,1 Afnan Al-Ali,2 and Turki Abdalla2, "A New Chaos-Based Image-Encryption and Compression Algorithm" Journal of Electrical and Computer Engineering Volume 2012, Article ID 179693, 11 pages.

[26] Hazem Mohammad Al-Najjar and Asem Mohammad AL-Najjar "Multi-Chaotic Image Encryption Algorithm Based on One Time Pads Scheme" International Journal of Computer Theory and Engineering, Vol. 4, No. 3, June 2012.

[27] F. K. Tabash, M.Q. Rafiq, M. Izharrudin, "Image Encryption Algorithm based on Chaotic Map "International Journal of Computer Applications (0975 - 8887) Volume 64- No.13, February 2013.

[28] Amit Gupta, Chandrashekhar Kamargaonkar, Monisha Sharma "Image EncryptionBased On Nonlinear Chaotic Algorithm" International Journal of Advanced Research in Electronics and Communication Engineering (IJARECE) Volume 2, Issue 6, June 2013.

[29] Chong Fu 1, Jun-Bin Huang, Ning-Ning Wang 1, Qi-Bin Hou 1 and Wei-Min Lei 1, "A Symmetric Chaos-Based Image Cipher with an Improved Bit-Level Permutation Strategy”, Entropy 2014, 16, 770-788.

[30] Muhammad Asif Gondal1 and Iqtadar Hussain2, "An Image Encryption Scheme based on Nonlinear Chaotic Algorithm and Substitution Box Transformation” Appl. Math. Inf. Sci. 9, No. 6, 2991-2995 (2015).

[31] Usha Salagundi1 , Nilam Chheda2, Kiran3, Usha Salagundi et al, "Image Encryption Using Scrambling and Diffusion Operation Using Chaotic Map" International Journal of Computer Science and Mobile Computing, Vol.5 Issue.5, May- 2016, pg. 343-348

[32] Wei Wang,1 Haiyan Tan,1 Yu Pang,1 Zhangyong Li,1 Peng Ran,1 and Jun Wu2, "A Novel Encryption Algorithm Based on DWT and Multichaos Mapping" Hindawi Publishing Corporation Journal of Sensors Volume 2016, Article ID 2646205, 7 pages.

[33] Srinivas koppu, madhu viswanatham v, “2D chaotic map based on 2D adaptive Grey Wolf Algorithm for Ultra Sound Medical Image Security", International Journal of Intelligent Engineering and Systems, Vol.10, No.1, 2017.

[34] Xiao Chen, Chun-Jie Hu "Medical image encryption based on multiple chaotic mapping and wavelet transform" Biomedical Research $2017 ; 28(20)$.

\section{AUTHOR PROFILE}

Dr. BANAVATH DHANALAXMI, B.Tech (ECE), M.Tech (VLSI System Design), Ph.D (ECE). Working as Assistant Professor, Department of Electronics and Communication Engineering, Kakatiya Institute of Technology and Science. My Ph. D was completed in faculty of Electronics and Communication Engineering from Kakatiya University, Telangana, India, under the supervision of Prof. Tadisetty Srinivasulu. I am interested to doing research in medical images security and Bio-medical sensors. 